\title{
Dirac-Deng-Fan Problem with Coulomb-Hulthen Tensor Interactions
}

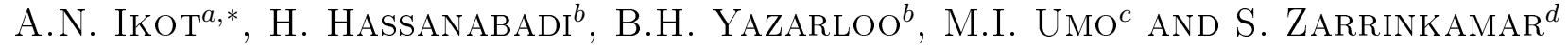 \\ ${ }^{a}$ Theoretical Physics Group, Department of Physics, University of Port Harcourt, \\ Choba, PMB 5323, Port Harcourt, Nigeria \\ ${ }^{b}$ Department of Basic Sciences, Shahrood Branch, Islamic Azad University, Shahrood, Iran \\ ${ }^{c}$ Department of Physics, University of Calabar, Nigeria \\ ${ }^{d}$ Department of Basic Sciences, Garmsar Branch, Islamic Azad University, Garmsar, Iran
}

(Received January 29, 2014; in final form April 2, 2014)

\begin{abstract}
The relativistic symmetries of the Dirac equation within the framework of spin and pseudospin symmetries is investigated for Deng-Fan potential including the Coulomb-like and Hulthen-type potential tensor interaction terms. The energy eigenvalues and the corresponding wave function are obtained using the parametric generalization of Nikiforov-Uvarov method. We have also reported some numerical results and figures to show the effect of the tensor interactions.
\end{abstract}

DOI: 10.12693/APhysPolA.126.656

PACS: 03.65.Ge, 03.65.Pm, 03.65.Db

\section{Introduction}

The Deng-Fan potential [1, 2] discovery more than 50 years ago is the simplest modified form of the Morse potential and is related to the Manning-Rosen and Eckart potentials. This potential is used to describe diatomic molecular energy spectra and electromagnetic transition and is usually regarded as the true internuclear potential in diatomic molecules [3-6]. In recent years there are lots of papers which discuss the spin and pseudospin symmetries in the Dirac equation with various potentials including Wood-Saxon, harmonic, Yukawa, hyperbolic and others [7-18]. The spin and pseudospin symmetries of the Dirac Hamiltonian had been discovered many years ago, however, these symmetries have recently been recognized empirically in nuclear and hadronic spectroscopy [19-22]. Within the theory of the Dirac equation, pseudospin symmetry is used to feature deformed nuclei and superdeformation to establish an effective shell model [23]. On the other hand, the spin symmetry is relevant for mesons [24].

It was shown that the exact pseudospin symmetry occurs in the Dirac equation when $\frac{\mathrm{d} \Sigma(r)}{\mathrm{d} r}=0$, i.e. $\Sigma(r)=V(r)+S(r)=c_{\mathrm{ps}}=$ const, where $V(r), S(r)$ are repulsive and attractive scalar potentials, respectively. On the other hand, the exact spin symmetry occurs in the Dirac equation when $\frac{\mathrm{d} \Delta(r)}{\mathrm{d} r}=0$, where $\Delta(r)=V(r)-S(r)=c_{\mathrm{s}}=$ const. $\stackrel{\text { The pseudospin }}{\mathrm{T}}$ symmetry usually refers to as a quasi-degeneracy of single nucleon doublets with non-relativistic quantum number $\left(n, l, j=l+\frac{1}{2}\right)$ and $\left(n-1, l+2, j=l+\frac{3}{2}\right)$, where $n, l$, and $j$ are single nucleon radial, orbital, and total angular quantum numbers, respectively. The total angular momentum is $j=\tilde{l}+\tilde{s}$, where $\tilde{l}=l+1$ is a

* corresponding author; e-mail: ndemikotphysics@gmail.com pseudo-angular momentum and $\tilde{s}$ is pseudospin angular momentum. Similarly, the tensor interaction term was introduced into the Dirac equation with the replacement $\boldsymbol{p} \rightarrow \boldsymbol{p}-\mathrm{i} M \omega \beta \cdot \hat{r} U(r)$ and a spin-orbit coupling is added to the Dirac Hamiltonian [25].

\section{The Nikiforov-Uvarov method}

The NU method can solve a second-order differential equation of the form [26-31]:

$$
\psi_{n}^{\prime \prime}(s)+\frac{\tilde{\tau}(s)}{\sigma(s)} \psi_{n}^{\prime}(s)+\frac{\tilde{\sigma}(s)}{\sigma^{2}(s)} \psi_{n}(s)=0,
$$

where $\sigma(s)$ and $\tilde{\sigma}(s)$ are polynomials, at most of second degree, and $\tilde{\tau}(s)$ is a first-degree polynomial. To make the application of the NU method simpler and more direct, we introduce a more compact presentation of the idea. In order to do this, we rewrite Eq. (1) as follows:

$$
\begin{aligned}
& \psi_{n}^{\prime \prime}(s)+\frac{c_{1}-c_{2} s}{s\left(1-c_{3} s\right)} \psi_{n}^{\prime}(s) \\
& +\frac{-\xi_{1} s^{2}+\xi_{2} s-\xi_{3}}{s^{2}\left(1-c_{3} s\right)^{2}} \psi_{n}(s)=0,
\end{aligned}
$$

in which

$$
\psi_{n}(s)=\varphi(s) y_{n}(s) .
$$

Comparing Eq. (2) with Eq. (3), we obtain the following identifications:

$$
\begin{aligned}
& \tilde{\tau}(s)=c_{1}-c_{2} s, \quad \sigma(s)=s\left(1-c_{3} s\right), \\
& \tilde{\sigma}(s)=-\xi_{1} s^{2}+\xi_{2} s-\xi_{3} .
\end{aligned}
$$

Following the NU method, we obtain the following required parameters:

(i) the relevant constant

$$
\begin{aligned}
& c_{4}=\frac{1}{2}\left(1-c_{1}\right), \quad c_{5}=\frac{1}{2}\left(c_{2}-2 c_{3}\right), \\
& c_{6}=c_{5}^{2}+\xi_{1}, \quad c_{7}=2 c_{4} c_{5}-\xi_{2}, \\
& c_{8}=c_{4}^{2}+\xi_{3}, \quad c_{9}=c_{3} c_{7}+c_{3}^{2} c_{8}+c_{6}, \\
& c_{10}=c_{1}+2 c_{4}+2 \sqrt{c_{8}}, \\
& c_{11}=c_{2}-2 c_{5}+2\left(\sqrt{c_{9}}+c_{3} \sqrt{c_{8}}\right),
\end{aligned}
$$


$c_{12}=c_{4}+\sqrt{c_{8}}, \quad c_{13}=c_{5}-\left(\sqrt{c_{9}}+c_{3} \sqrt{c_{8}}\right)$;

(ii) the essential polynomial functions

$$
\begin{aligned}
& \pi(s)=c_{4}+c_{5} s-\left[\left(\sqrt{c_{9}}+c_{3} \sqrt{c_{8}}\right) s-\sqrt{c_{8}}\right], \\
& k=-\left(c_{7}+2 c_{3} c_{8}\right)-2 \sqrt{c_{8} c_{9}}, \\
& \tau(s)=c_{1}+2 c_{4}-\left(c_{2}-2 c_{5}\right) s \\
& \quad-2\left[\left(\sqrt{c_{9}}+c_{3} \sqrt{c_{8}}\right) s-\sqrt{c_{8}}\right], \\
& \tau^{\prime}(s)=-2 c_{3}-2\left(\sqrt{c_{9}}+c_{3} \sqrt{c_{8}}\right)<0
\end{aligned}
$$

(iii) the energy equation

$$
\begin{aligned}
& c_{2} n-(2 n+1) c_{5}+(2 n+1)\left(\sqrt{c_{9}}+c_{3} \sqrt{c_{8}}\right) \\
& \quad+n(n-1) c_{3}+c_{7}+2 c_{3} c_{8}+2 \sqrt{c_{8} c_{9}}=0 ;
\end{aligned}
$$

(iv) the wave functions

$$
\begin{aligned}
& \rho(s)=s^{c_{10}}\left(1-c_{3} s\right)^{c_{11}}, \\
& \varphi(s)=s^{c_{12}}\left(1-c_{3} s\right)^{c_{13}}, \quad c_{12}>0, c_{13}>0, \\
& y_{n}(s)=P_{n}^{\left(c_{10}, c_{11}\right)}\left(1-2 c_{3} s\right), \\
& c_{10}>-1, \quad c_{11}>-1, \\
& \psi_{n \kappa}(s)=N_{n \kappa} s^{c_{12}}\left(1-c_{3} s\right)^{-c_{12}-\frac{c_{13}}{c_{3}}} \\
& \quad \times P_{n}^{\left(c_{10}-1, \frac{c_{11}}{c_{3}}-c_{10}-1\right)}\left(1-2 c_{3} s\right),
\end{aligned}
$$

where $P_{n}^{(\mu, \nu)}(x), \mu>-1, \nu>-1$, and $x \in[-1,1]$ are the Jacobi polynomials with

$$
\begin{aligned}
& P_{n}^{(\alpha, \beta)}(1-2 s)=\frac{(\alpha+1)_{n}}{n !} \\
& \quad \times{ }_{2} F_{1}(-n, 1+\alpha+\beta+n ; \alpha+1 ; s),
\end{aligned}
$$

and $N_{n \kappa}$ is a normalization constant. Also, the above wave functions can be expressed in terms of the hypergeometric function via

$$
\begin{aligned}
& \psi_{n \kappa}(s)=N_{n \kappa} s^{c_{12}}\left(1-c_{3} s\right)^{c_{13}} \\
& \quad \times{ }_{2} F_{1}\left(-n, 1+c_{10}+c_{11}+n ; c_{10}+1 ; c_{3} s\right),
\end{aligned}
$$

where $c_{12}>0, c_{13}>0$ and $s \in\left[0,1 / c_{3}\right], c_{3} \neq 0$.

\section{Theory of Dirac equation}

The Dirac equation for spin $\frac{1}{2}$ particles moving in an attractive scalar potential $S(r)$, a repulsive vector potential $V(r)$ and a tensor potential $U(r)$ in the relativistic unit $(\hbar=c=1)$ is

$$
\begin{aligned}
& {[\boldsymbol{\alpha} \cdot \boldsymbol{p}+\beta(M+S(r))-\mathrm{i} \beta \boldsymbol{\alpha} \cdot \hat{r} U(r)] \psi(r)} \\
& \quad=[E-V(r)] \psi(r),
\end{aligned}
$$

where $E$ is the relativistic energy of the system, $\boldsymbol{p}=-\mathrm{i} \nabla$ is the three-dimensional momentum operator and $M$ is the mass of the fermionic particle. $\alpha, \beta$ are the $4 \times 4$ Dirac matrices given as

$$
\boldsymbol{\alpha}=\left(\begin{array}{cc}
0 & \boldsymbol{\sigma}_{i} \\
\boldsymbol{\sigma}_{i} & 0
\end{array}\right), \quad \beta=\left(\begin{array}{cc}
I & 0 \\
0 & -I
\end{array}\right),
$$

where $I$ is $2 \times 2$ unitary matrix and $\sigma_{i}$ are the Pauli three-vector matrices

$$
\sigma_{1}=\left(\begin{array}{ll}
0 & 1 \\
1 & 0
\end{array}\right), \quad \sigma_{2}=\left(\begin{array}{cc}
0 & -\mathrm{i} \\
\mathrm{i} & 0
\end{array}\right), \quad \sigma_{3}=\left(\begin{array}{cc}
1 & 0 \\
0 & -1
\end{array}\right) .
$$

The eigenvalues of the spin-orbit coupling operator are $\kappa=\left(j+\frac{1}{2}\right) \succ 0, \kappa=-\left(j+\frac{1}{2}\right) \prec 0$ for unaligned $j=l-\frac{1}{2}$ and the aligned spin $j=l+\frac{1}{2}$, respectively. The set $\left(H, K, J^{2}, J_{z}\right)$ forms a complete set of conserved quantities. Thus, we can write the spinors as

$$
\psi_{n \kappa}(r)=\frac{1}{r}\left(\begin{array}{l}
F_{n \kappa}(r) Y_{j m}^{l}(\theta, \varphi) \\
\mathrm{i} G_{n \kappa}(r) Y_{j m}^{\tilde{l}}(\theta, \varphi)
\end{array}\right),
$$

where $F_{n \kappa}(r), G_{n \kappa}(r)$ represent the upper and lower components of the Dirac spinors. $Y_{j m}^{l}(\theta, \varphi), Y_{j m}^{\tilde{l}}(\theta, \varphi)$ are the spin and pseudospin spherical harmonics and $m$ is the projection on the $z$-axis. Using well-known identities

$$
\begin{aligned}
& (\boldsymbol{\sigma} \cdot \boldsymbol{A})(\boldsymbol{\sigma} \cdot \boldsymbol{B})=\boldsymbol{A} \cdot \boldsymbol{B}+\mathrm{i} \boldsymbol{\sigma} \cdot(\boldsymbol{A} \times \boldsymbol{B}), \\
& \boldsymbol{\sigma} \cdot \boldsymbol{p}=\boldsymbol{\sigma} \cdot \hat{r}\left(\hat{r} \cdot \boldsymbol{p}+\mathrm{i} \frac{\boldsymbol{\sigma} \cdot \boldsymbol{L}}{r}\right),
\end{aligned}
$$

as well as the relations

$$
\begin{aligned}
& (\boldsymbol{\sigma} \cdot \boldsymbol{L}) Y_{j m}^{\tilde{l}}(\theta, \varphi)=(\kappa-1) Y_{j m}^{\tilde{l}}(\theta, \varphi), \\
& (\boldsymbol{\sigma} \cdot \boldsymbol{L}) Y_{j m}^{l}(\theta, \varphi)=-(\kappa-1) Y_{j m}^{l}(\theta, \varphi), \\
& (\boldsymbol{\sigma} \cdot \hat{r}) Y_{j m}^{l}(\theta, \varphi)=-Y_{j m}^{\tilde{l}}(\theta, \varphi), \\
& (\boldsymbol{\sigma} \cdot \hat{r}) Y_{j m}^{\tilde{l}}(\theta, \varphi)=-Y_{j m}^{l}(\theta, \varphi),
\end{aligned}
$$

we find the following two coupled first-order Dirac equation:

$$
\begin{aligned}
& \left(\frac{\mathrm{d}}{\mathrm{d} r}+\frac{\kappa}{r}-U(r)\right) F_{n \kappa}(r) \\
& \quad=\left(M+E_{n \kappa}-\Delta(r)\right) G_{n \kappa}(r), \\
& \left(\frac{\mathrm{d}}{\mathrm{d} r}-\frac{\kappa}{r}+U(r)\right) G_{n \kappa}(r) \\
& \quad=\left(M-E_{n \kappa}+\Sigma(r)\right) F_{n \kappa}(r),
\end{aligned}
$$

where

$$
\begin{aligned}
& \Delta(r)=V(r)-S(r), \\
& \Sigma(r)=V(r)+S(r) .
\end{aligned}
$$

Eliminating $F_{n \kappa}(r)$ and $G_{n \kappa}(r)$ in Eqs. (23) and (24), we obtain the second-order Schrödinger-like equation

$$
\begin{aligned}
& \left\{\frac{\mathrm{d}^{2}}{\mathrm{~d} r^{2}}-\frac{\kappa(\kappa+1)}{r^{2}}+\frac{2 \kappa U(r)}{r}-\frac{\mathrm{d} U(r)}{\mathrm{d} r}-U^{2}(r)\right. \\
& -\left[M+E_{n \kappa}-\Delta(r)\right]\left[M-E_{n \kappa}+\Sigma(r)\right] \\
& \left.+\frac{\frac{\mathrm{d} \Delta(r)}{\mathrm{d} r}\left(\frac{\mathrm{d}}{\mathrm{d} r}+\frac{\kappa}{r}-U(r)\right)}{M+E_{n \kappa}-\Delta(r)}\right\} F_{n \kappa}(r)=0, \\
& \left\{\frac{\mathrm{d}^{2}}{\mathrm{~d} r^{2}}-\frac{\kappa(\kappa-1)}{r^{2}}+\frac{2 \kappa U(r)}{r}+\frac{\mathrm{d} U(r)}{\mathrm{d} r}-U^{2}(r)\right. \\
& -\left[M+E_{n \kappa}-\Delta(r)\right]\left[M-E_{n \kappa}+\Sigma(r)\right] \\
& \left.+\frac{\frac{\mathrm{d} \Sigma(r)}{\mathrm{d} r}\left(\frac{\mathrm{d}}{\mathrm{d} r}-\frac{\kappa}{r}+U(r)\right)}{M-E_{n \kappa}+\Sigma(r)}\right\} G_{n \kappa}(r)=0,
\end{aligned}
$$

where $\kappa(\kappa-1)=\tilde{l}(\tilde{l}+1), \kappa(\kappa+1)=l(l+1)$. 


\section{Pseudospin and spin symmetry limits}

In this section, we intend to investigate the the Dirac equation with Deng-Fan potential in the presence of the Coulomb-Hulthen tensor interactions.

\subsection{Pseudospin symmetry in the Dirac equation}

The pseudospin symmetry occurs in the Dirac equation when $\frac{\mathrm{d} \Sigma(r)}{\mathrm{d} r}=0$ or equivalently $\Sigma(r)=C_{\mathrm{ps}}=$ const. In order to investigate the approximate analytical solution of the Deng-Fan potential, we consider the sum of the scalar and vector potential as [1],

$$
\Delta(r)=D\left(1-\frac{b}{\mathrm{e}^{\eta r}-1}\right)^{2}, \quad b=\mathrm{e}^{\eta r_{\mathrm{c}}},
$$

where $r_{c}$ is the distance from the equilibrium position. In addition, we proposed a novel generalized tensor interaction of the form

$$
U(r)=-\left(U_{\mathrm{C}}(r)+U_{\mathrm{H}}(r)\right),
$$

where $U_{\mathrm{C}}(r)$ and $U_{\mathrm{H}}(r)$ are the Coulomb-like and Hulthen-like potentials [32] defined as

$$
\begin{aligned}
U_{\mathrm{C}}(r) & =-\frac{H_{\mathrm{c}}}{r}, \\
U_{\mathrm{H}}(r) & =-V_{\mathrm{H}} \frac{\mathrm{e}^{-\eta r}}{1-\mathrm{e}^{-\eta r}}
\end{aligned}
$$

with

$$
H_{\mathrm{c}}=\frac{z_{a} z_{b} e^{2}}{4 \pi \varepsilon_{0}},
$$

where $R_{\mathrm{e}}$ is the Coulomb radius, $z_{a}$ and $z_{b}$ denote the charges of the projectile $a$ and the target nuclei $b$ and $V_{\mathrm{H}}$ is the depth of the Hulthen potential. Substituting Eq. (32) into Eq. (31), we obtained our proposed GTI as

$$
U(r)=-\frac{H_{\mathrm{c}}}{r}-V_{\mathrm{H}} \frac{\mathrm{e}^{-\eta r}}{1-\mathrm{e}^{-\eta r}} .
$$

Substituting the above equations into Eq. (28) yields

$$
\begin{aligned}
& \left\{\frac{\mathrm{d}^{2}}{\mathrm{~d} r^{2}}-\frac{\kappa(\kappa-1)}{r^{2}}-\frac{2 \kappa H_{\mathrm{c}}}{r^{2}}+\frac{H_{\mathrm{c}}}{r^{2}}-\frac{H_{\mathrm{c}}^{2}}{r^{2}}\right. \\
& \left.\quad-\frac{2 \kappa V_{\mathrm{H}} \mathrm{e}^{-\eta r}}{r\left(1-\mathrm{e}^{-\eta r}\right)}-\frac{2 H_{\mathrm{c}} V_{\mathrm{H}} \mathrm{e}^{-\eta r}}{r\left(1-\mathrm{e}^{-\eta r}\right)}-\frac{V_{\mathrm{H}}^{2} \mathrm{e}^{-2 \eta r}}{\left(1-\mathrm{e}^{-\eta r}\right)^{2}}\right\} \\
& \quad \times G_{n, \kappa}^{\mathrm{ps}}(r)+\left[\frac{\eta V_{\mathrm{H}} \mathrm{e}^{-\eta r}}{1-\mathrm{e}^{-\eta r}}-\varepsilon_{\mathrm{ps}}^{2}\right. \\
& \left.\quad+\beta_{\mathrm{ps}}\left(1-\frac{b \mathrm{e}^{-\eta r}}{1-\mathrm{e}^{-\eta r}}\right)^{2}\right] G_{n \kappa}^{\mathrm{ps}}(r)=0,
\end{aligned}
$$

where

$$
\begin{aligned}
& \varepsilon_{\mathrm{ps}}^{2}=M^{2}+M C_{\mathrm{ps}}-\left(E_{n \kappa}^{\mathrm{ps}}\right)^{2}+C_{\mathrm{ps}} E_{n \kappa}^{\mathrm{ps}}, \\
& \beta_{\mathrm{ps}}=D\left(M-E_{n \kappa}^{\mathrm{ps}}+C_{\mathrm{ps}}\right) .
\end{aligned}
$$

It is well known that the above equation cannot be solved exactly due to the centrifugal term $r^{-2}$. In order to get rid of the centrifugal term, we make use of the following approximation [33-36] and the references therein

$$
\frac{1}{r^{2}} \approx \frac{\eta^{2} \mathrm{e}^{-2 \eta r}}{\left(1-\mathrm{e}^{-\eta r}\right)^{2}}, \quad \frac{1}{r} \approx \frac{\eta \mathrm{e}^{-\eta r}}{1-\mathrm{e}^{-\eta r}} .
$$

Substituting Eqs. (37) into Eq. (34) in view of the transformation, $z=\mathrm{e}^{-\eta r}$, yields

$$
\begin{aligned}
& \frac{\mathrm{d}^{2} G_{n, \kappa}^{\mathrm{ps}}}{\mathrm{d} z^{2}}+\frac{1-z}{z(1-z)} \frac{\mathrm{d} G_{n, \kappa}^{\mathrm{ps}}}{\mathrm{d} z}+\frac{1}{z^{2}(1-z)^{2}} \\
& \quad \times\left[-L^{\mathrm{ps}} z^{2}+K^{\mathrm{ps}} z-f^{\mathrm{ps}}\right] G_{n, \kappa}^{\mathrm{ps}}(z)=0,
\end{aligned}
$$

where

$$
\begin{aligned}
L^{\mathrm{ps}} & =\eta_{\kappa}\left(\eta_{\kappa}-1\right)+\frac{2 \kappa V_{\mathrm{H}}}{\eta}+\frac{2 H_{\mathrm{c}} V_{\mathrm{H}}}{\eta}+\frac{V_{\mathrm{H}}^{2}}{\eta^{2}} \\
& -\frac{1}{\eta^{2}}\left(D b^{2}+2 b D-M-E_{n \kappa}^{\mathrm{ps}}+D\right) \\
& \times\left(M-E_{n \kappa}^{\mathrm{ps}}+C_{\mathrm{ps}}\right), \\
K^{\mathrm{ps}} & =\frac{V_{\mathrm{H}}}{\eta}+\frac{2}{\eta^{2}}\left(-b D+M+E_{n \kappa}^{\mathrm{ps}}-D\right) \\
& \times\left(M-E_{n \kappa}^{\mathrm{ps}}+C_{\mathrm{ps}}\right), \\
f^{\mathrm{ps}} & =\frac{1}{\eta^{2}}\left(M+E_{n \kappa}^{\mathrm{ps}}-D\right)\left(M-E_{n \kappa}^{\mathrm{ps}}+C_{\mathrm{ps}}\right), \\
H_{\mathrm{c}}^{2} & +2 \kappa H_{\mathrm{c}}+\kappa(\kappa-1)-H_{\mathrm{c}}=\eta_{\kappa}\left(\eta_{\kappa}-1\right) \\
& \rightarrow \eta_{\kappa}=\kappa+H_{\mathrm{c}} .
\end{aligned}
$$

\subsection{Spin symmetry in the Dirac equation}

In the spin symmetry limit condition, we take the sum potential $\Sigma(r)$ as the Deng-Fan potential, the difference potential $\Delta(r)$ as constant and the tensor potential $U(r)$ as the Coulomb-Hulthen term. Thus, we have the following:

$$
\begin{aligned}
& \Sigma(r)=D\left(1-\frac{b}{\mathrm{e}^{\eta r}-1}\right)^{2}, \quad \Delta(r)=C_{s}, \\
& U(r)=-\frac{1}{r}\left(H_{\mathrm{c}}+V_{\mathrm{H}} \frac{\mathrm{e}^{-\eta r}}{1-\mathrm{e}^{-\eta r}}\right) .
\end{aligned}
$$

Substituting Eq. (43) into Eq. (27) yields

$$
\begin{aligned}
& \left\{\frac{\mathrm{d}^{2}}{\mathrm{~d} r^{2}}-\frac{\kappa(\kappa+1)}{r^{2}}-\frac{2 \kappa H_{\mathrm{c}}}{r^{2}}-\frac{H_{\mathrm{c}}}{r^{2}}-\frac{H_{\mathrm{c}}^{2}}{r^{2}}\right. \\
& \quad-\left(M+E_{n \kappa}-C_{s}\right)\left(M-E_{n \kappa}\right)-\left(M+E_{n \kappa}-C_{s}\right) \\
& \left.\quad \times D\left(1-\frac{b \mathrm{e}^{-\eta r}}{1-\mathrm{e}^{-\eta r}}\right)^{2}\right\} F_{n, \kappa}^{s}(r)-\left(\frac{2 \kappa V_{\mathrm{H}} \mathrm{e}^{-\eta r}}{r\left(1-\mathrm{e}^{-\eta r}\right)}\right. \\
& \left.\quad+\frac{V_{\mathrm{H}} \eta \mathrm{e}^{-\eta r}}{1-\mathrm{e}^{-\eta r}}+\frac{2 H_{\mathrm{c}} V_{\mathrm{H}} \mathrm{e}^{-\eta r}}{r\left(1-\mathrm{e}^{-\eta r}\right)}+\frac{V_{\mathrm{H}}^{2} \mathrm{e}^{-2 \eta r}}{\left(1-\mathrm{e}^{-\eta r}\right)^{2}}\right) \\
& \quad \times F_{n \kappa}^{s}(r)=0 .
\end{aligned}
$$

By using the approximation of Eq. (37) for the centrifugal term in Eq. (44), then Eq. (44) leads to the following second-order differential equation in view of the $s=\mathrm{e}^{-\eta r}$ transformation,

$$
\begin{gathered}
\frac{\mathrm{d}^{2} F_{n, \kappa}^{s}}{\mathrm{~d} s^{2}}+\frac{1-s}{s(1-s)} \frac{\mathrm{d} F_{n, \kappa}^{s}}{\mathrm{~d} s}+\frac{1}{s^{2}(1-s)^{2}} \\
\times\left[-\mu^{s} s^{2}+\chi^{s} s-g^{s}\right] F_{n, \kappa}^{s}(s)=0,
\end{gathered}
$$

where 


$$
\begin{aligned}
\mu^{s} & =\Lambda_{\kappa}\left(\Lambda_{\kappa}-1\right)+\frac{2 \kappa V_{\mathrm{H}}}{\eta}+\frac{2 H_{\mathrm{c}} V_{\mathrm{H}}}{\eta}+\frac{V_{\mathrm{H}}^{2}}{\eta^{2}} \\
& +\frac{1}{\eta^{2}}\left(D b^{2}+2 b D+M-E_{n \kappa}^{s}+D\right) \\
& \times\left(M+E_{n \kappa}^{s}-C_{s}\right), \\
\chi^{s} & =-\frac{V_{\mathrm{H}}}{\eta}+\frac{2}{\eta^{2}}\left(b D+M-E_{n \kappa}^{s}+D\right) \\
& \times\left(M+E_{n \kappa}^{s}-C_{s}\right), \\
g^{s} & =\frac{1}{\eta^{2}}\left(M-E_{n \kappa}^{s}+D\right)\left(M+E_{n \kappa}^{s}-C_{s}\right), \\
H_{\mathrm{c}}^{2} & +2 \kappa H_{\mathrm{c}}+\kappa(\kappa-1)+H_{\mathrm{c}}=\Lambda_{\kappa}\left(\Lambda_{\kappa}-1\right) \\
& \rightarrow \Lambda_{\kappa}=\left(\kappa+H_{\mathrm{c}}+1\right) .
\end{aligned}
$$

\subsection{Pseudospin and spin symmetry solutions}

We will solve the solutions of Eqs. (39) and (45) by using the parametric generalization of the NU method in the subsequent section.

\subsection{Pseudospin symmetry solution}

Now comparing Eq. (2) with Eq. (39), we obtain

$$
\begin{aligned}
& c_{1}=1, \quad c_{2}=1, \quad c_{3}=1, \\
& \xi_{1}=L^{\mathrm{ps}}, \quad \xi_{2}=K^{\mathrm{ps}}, \quad \xi_{3}=f^{\mathrm{ps}} .
\end{aligned}
$$

Other parameters can be obtained from Eq. (5) as

$$
\begin{aligned}
& c_{4}=0, \quad c_{5}=-\frac{1}{2}, \quad c_{6}=\frac{1}{4}+L^{\mathrm{ps}}, \quad c_{7}=-K^{\mathrm{ps}}, \\
& c_{8}=f^{\mathrm{ps}}, \quad c_{9}=\frac{1}{4}+L^{\mathrm{ps}}+f^{\mathrm{ps}}-K^{\mathrm{ps}}, \\
& c_{10}=1+2 \sqrt{f^{\mathrm{ps}}} \\
& c_{11}=2+2\left[\sqrt{\frac{1}{4}+L^{\mathrm{ps}}+f^{\mathrm{ps}}-K^{\mathrm{ps}}}+\sqrt{f^{\mathrm{ps}}}\right], \\
& c_{12}=\sqrt{f^{\mathrm{ps}}}, \\
& c_{13}=-\frac{1}{2}-\left(\sqrt{\frac{1}{4}+L^{\mathrm{ps}}+f^{\mathrm{ps}}-K^{\mathrm{ps}}}+\sqrt{f^{\mathrm{ps}}}\right) .
\end{aligned}
$$

Substituting Eqs. (50) and (51) into Eq. (10) yields

$$
\begin{aligned}
n^{2} & +\left(n+\frac{1}{2}\right)+(2 n+1) \\
& \times\left[\sqrt{\frac{1}{4}+L^{\mathrm{ps}}+f^{\mathrm{ps}}-L^{\mathrm{ps}}}+\sqrt{f^{\mathrm{ps}}}\right]-K^{\mathrm{ps}}+2 f^{\mathrm{ps}} \\
& +2 \sqrt{f^{\mathrm{ps}}\left(\frac{1}{4}+L^{\mathrm{ps}}+f^{\mathrm{ps}}-K^{\mathrm{ps}}\right)}=0 .
\end{aligned}
$$

From Eqs. (14) and (15) the lower components of the wave functions are as

$$
\begin{aligned}
& G_{n, \kappa}^{\mathrm{ps}}(r)=N_{n, \kappa}^{\mathrm{ps}} \mathrm{e}^{-\eta \sqrt{\rho_{3}^{\mathrm{ps}}} r} \\
& \times\left(1-\mathrm{e}^{-\eta r}\right)^{\frac{1}{2}+\sqrt{\frac{1}{4}+L^{\mathrm{ps}}+f^{\mathrm{ps}}-K^{\mathrm{ps}}}} \\
& \times P_{n}^{\left(2 \sqrt{f^{\mathrm{ps}}}, 2 \sqrt{\frac{1}{4}+L^{\mathrm{ps}}+f^{\mathrm{ps}}-K^{\mathrm{ps}}}\right)}\left(1-2 \mathrm{e}^{-\eta r}\right)
\end{aligned}
$$

obtained as

$$
\begin{aligned}
& F_{n, \kappa}^{\mathrm{ps}}(r)=\frac{1}{M-E_{n, \kappa}^{\mathrm{ps}}+C_{\mathrm{ps}}}\left(\frac{\mathrm{d}}{\mathrm{d} r}-\frac{\kappa}{r}+U(r)\right) \\
& \quad \times G_{n, \kappa}^{\mathrm{ps}}(r),
\end{aligned}
$$

where $N_{n, \kappa}^{\mathrm{ps}}$ is the normalization constant and $E_{n, \kappa}^{\mathrm{ps}} \neq$ $M+C_{\mathrm{ps}}$.

\subsection{Spin symmetry solution}

In order to avoid repetition, we applied the same procedure for the spin symmetry limits. The energy eigenvalues equation and the corresponding upper wave function of the Dirac theory for the hyperbolical potential in the presence of generalized tensor interaction are obtained as

$$
\begin{aligned}
& n^{2}+\left(n+\frac{1}{2}\right)+(2 n+1) \\
& \quad \times\left[\sqrt{\frac{1}{4}+\mu^{s}+g^{s}-\chi^{s}}+\sqrt{g^{s}}\right]-\chi^{s}+2 g^{s} \\
& \quad+2 \sqrt{g^{s}\left(\frac{1}{4}+\mu^{s}+g^{s}-\chi^{s}\right)}=0, \\
& F_{n, \kappa}^{s}(r)=N_{n, \kappa}^{s} \mathrm{e}^{-\eta \sqrt{g^{s}} r}\left(1-\mathrm{e}^{-\eta r}\right)^{\frac{1}{2}+\sqrt{\frac{1}{4}+\mu^{s}+g^{s}-\chi^{s}}} \\
& \quad \times P_{n}^{\left(2 \sqrt{g^{s}}, 2 \sqrt{\frac{1}{4}+\mu^{s}+g^{s}-\chi^{s}}\right)}\left(1-2 \mathrm{e}^{-\eta r}\right),
\end{aligned}
$$

where $N_{n, \kappa}^{s}$ is the normalization constant.

The other component of the Dirac spinor can be found as

$$
\begin{aligned}
& G_{n, \kappa}^{s}(r)=\frac{1}{M+E_{n, \kappa}^{s}-C_{s}}\left(\frac{\mathrm{d}}{\mathrm{d} r}+\frac{\kappa}{r}-U(r)\right) \\
& \quad \times F_{n, \kappa}^{s}(r) .
\end{aligned}
$$

\section{Discussion and numerical results}

From Eq. (52) and Eq. (55), we have calculated the energy of the symmetry limits and reported in Tables I and II where we can see degeneracies between the states in the absence of tensor interaction, in the states $\left(1 s_{1 / 2}, 0 d_{3 / 2}\right),\left(1 p_{3 / 2}, 0 f_{5 / 2}\right), \ldots$ in pseudospin symmetry and the states $\left(0 p_{1 / 2}, 0 p_{3 / 2}\right),\left(0 d_{3 / 2}, 0 d_{5 / 2}\right) \ldots$ in spin symmetry limit. When $H_{\mathrm{c}}=V_{\mathrm{H}}=0.5$, these degeneracies disappear.

The effect of tensor interaction on the upper and lower components of the Dirac equation is represented in Figs. 1 and 2 for the symmetries.

To show the effect of parameter $\eta$ on the energy of the pseudospin and spin symmetries we have represented Fig. 3. It is obvious that as $\eta$ increases the energy of pseudospin (spin) symmetry decreases (increases). Figure 4 shows the behavior of the energy of the system versus $D$ for the symmetries. In the presence of tensor interaction, we have plotted the energy of the system vs. $b$ and $V_{\mathrm{H}}$ in Figs. 5 and 6 . It is seen in Fig. 5 that although pseudospin bound-states become less bounded with increasing $b$, the spin partners behave in a reverse way.

and the other component of the wave function can be 
TABLE I

Energy of the pseudospin symmetry limit in the presence and absence of Coulomb + Hulthen tensor interaction $M=5, \eta=0.1, D=-2, b=1, C_{\mathrm{ps}}=-5$.

\begin{tabular}{c|c|c|c|c|c|c}
\hline \hline$\tilde{l}$ & $n, k$ & State & $E_{n, k\left(H_{\mathrm{c}}=V_{\mathrm{H}}=0\right)}^{\mathrm{ps}}$ & $E_{n, k\left(H_{\mathrm{c}}=0, V_{\mathrm{H}}=0.5\right)}^{\mathrm{ps}}$ & $E_{n, k\left(H_{\mathrm{c}}=0.5, V_{\mathrm{H}}=0\right)}^{\mathrm{ps}}$ & $E_{n, k\left(H_{\mathrm{c}}=V_{\mathrm{H}}=0.5\right)}^{\mathrm{ps}}$ \\
\hline 1 & $1,-1$ & $1 s_{\frac{1}{2}}$ & -5.343164069 & -5.350270107 & -5.341275386 & -5.355815929 \\
2 & $1,-2$ & $1 p_{\frac{3}{2}}$ & -5.349171110 & -5.341296838 & -5.345799009 & -5.345425009 \\
3 & $1,-3$ & $1 d_{\frac{5}{2}}$ & -5.358078361 & -5.335244964 & -5.353268842 & -5.337899601 \\
4 & $1,-4$ & $1 f_{\frac{7}{2}}$ & -5.369766684 & -5.332197756 & -5.363583652 & -5.333342133 \\
1 & 0,2 & $0 d_{\frac{3}{2}}$ & -5.343164069 & -5.393356449 & -5.345799009 & -5.402665230 \\
2 & 0,3 & $0 f_{\frac{5}{2}}$ & -5.349171110 & -5.412514794 & -5.353268842 & -5.422877991 \\
3 & 0,4 & $0 g_{\frac{7}{2}}$ & -5.358078361 & -5.433727183 & -5.363583652 & -5.445034420 \\
4 & 0,5 & $0 h_{\frac{9}{2}}$ & -5.369766684 & -5.456771614 & -5.376607584 & -5.468910692
\end{tabular}

TABLE II

Energy of the spin symmetry limit in the presence and absence of Coulomb + Hulthen tensor interaction $M=5, \eta=0.1, d=2, b=2, C_{s}=5$.

\begin{tabular}{c|c|c|c|c|c|c}
\hline \hline$l$ & $n, k$ & State & $E_{n, k\left(H_{\mathrm{c}}=V_{\mathrm{H}}=0\right)}^{s}$ & $E_{n, k\left(H_{\mathrm{c}}=0, V_{\mathrm{H}}=0.5\right)}^{s}$ & $E_{n, k\left(H_{\mathrm{c}}=0.5, V_{\mathrm{H}}=0\right)}^{s}$ & $E_{n, k\left(H_{\mathrm{c}}=V_{\mathrm{H}}=0.5\right)}^{s}$ \\
\hline 1 & $0,-2$ & $0 p_{\frac{3}{2}}$ & 5.093710674 & 5.103048863 & 5.093126585 & 5.104779245 \\
2 & $0,-3$ & $0 d_{\frac{5}{2}}$ & 5.095576485 & 5.100271298 & 5.094527579 & 5.101545721 \\
3 & $0,-4$ & $0 f_{\frac{7}{2}}$ & 5.098365898 & 5.098413430 & 5.096856348 & 5.099226856 \\
4 & $0,-5$ & $0 g_{\frac{9}{2}}$ & 5.102067878 & 5.097482639 & 5.100103644 & 5.097831831 \\
1 & 0,1 & $0 p_{\frac{1}{2}}$ & 5.093710674 & 5.116773177 & 5.094527579 & 5.119824253 \\
2 & 0,2 & $0 d_{\frac{3}{2}}$ & 5.095576485 & 5.123086510 & 5.096856348 & 5.126556923 \\
3 & 0,3 & $0 f_{\frac{5}{2}}$ & 5.098365898 & 5.130232305 & 5.100103644 & 5.134109320 \\
4 & 0,4 & $0 g_{\frac{7}{2}}$ & 5.102067878 & 5.138184489 & 5.104256682 & 5.142454201
\end{tabular}

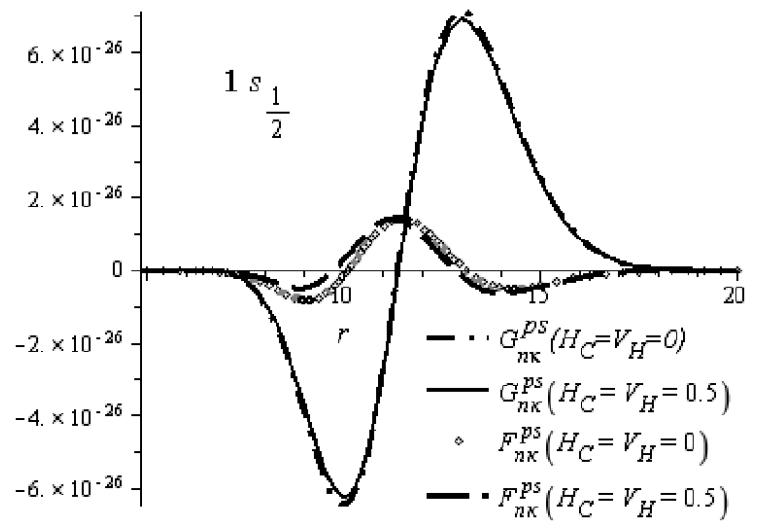

Fig. 1. Wave function of pseudospin symmetry for $M=5, \eta=0.1, d=-2, b=2, C_{\mathrm{ps}}=-5$.

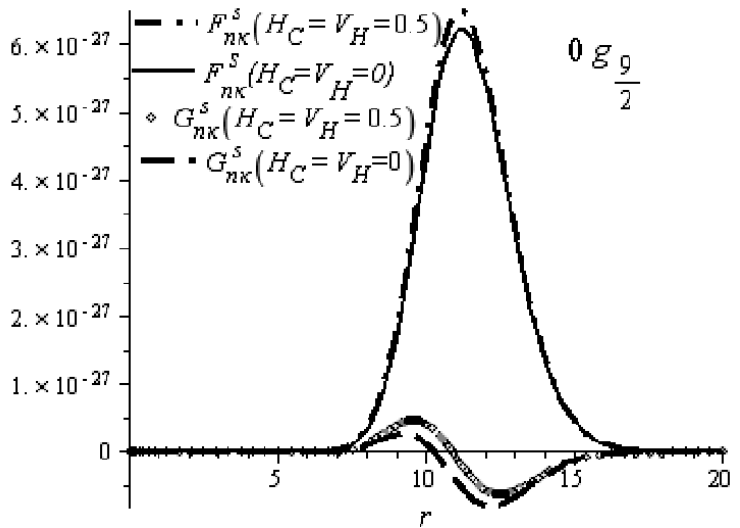

Fig. 2. Wave function of pseudospin symmetry for $M=5, \eta=0.1, d=2, b=2, C_{s}=5$. 


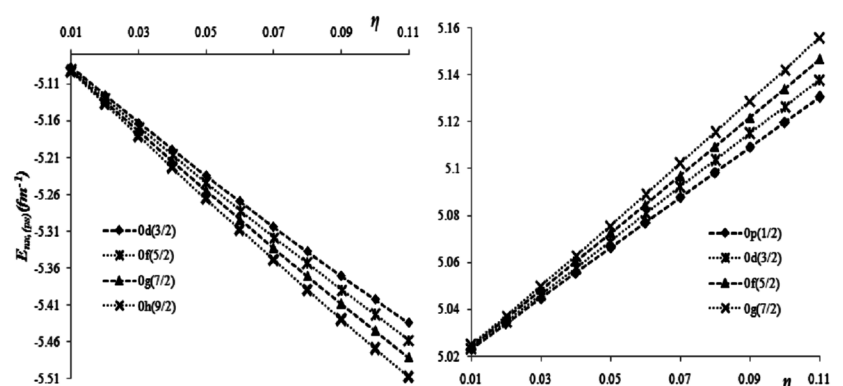

Fig. 3. Energy of the spin symmetry and pseudospin symmetry limits in the presence of Coulomb + Hulthen tensor interaction versus $\eta$.
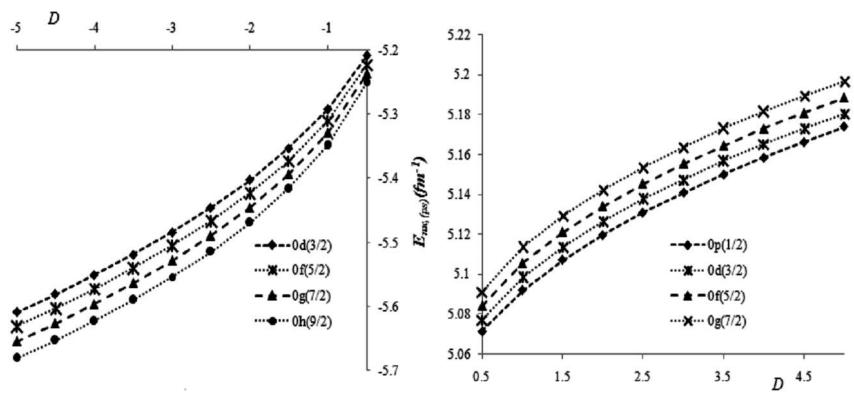

Fig. 4. Energy of the spin symmetry and pseudospin symmetry limits in the presence of Coulomb + Hulthen tensor interaction versus $D$.

\section{Conclusions}

In the present paper, we have obtained the approximate solutions of the Dirac equation for the Deng-Fan potential including the Coulomb-Hulthen tensor interactions in the framework of pseudospin and spin symmetry limits using the parametric generalization of NU method. We have obtained the energies eigenvalues and the corresponding lower and upper wave functions expressed in terms of the hypergeometric functions.

We have also computed the numerical results of our work and it shows that the presence of the combined Coulomb-Hulthen potentials remove the degeneracies between two states in spin and pseudospin doublets. To the best of our knowledge the Dirac equation with the Deng-Fan potential under the Coulomb-Hulthen tensor interactions had not been considered before using the NU method or any known method. Finally, the results of our work finds many applications in both nuclear, hadron and high energy physics.

\section{Acknowledgments}

It is a great pleasure for authors to thank the referee for his useful comments on the manuscript.

\section{References}

[1] Z.H. Deng, Y.P. Deng, Shandong Univ. J. 7, 162 (1957).

[2] S.H. Dong, X.Y. Gu, J. Phys., Conf. Series 96, 012109 (2008).

[3] B.H. Yazarloo, L. Lu, G. Liu, S. Zarrinkamar, H. Hassanabadi, Adv. High Energy Phys. 2013, ID 317605 (2013).

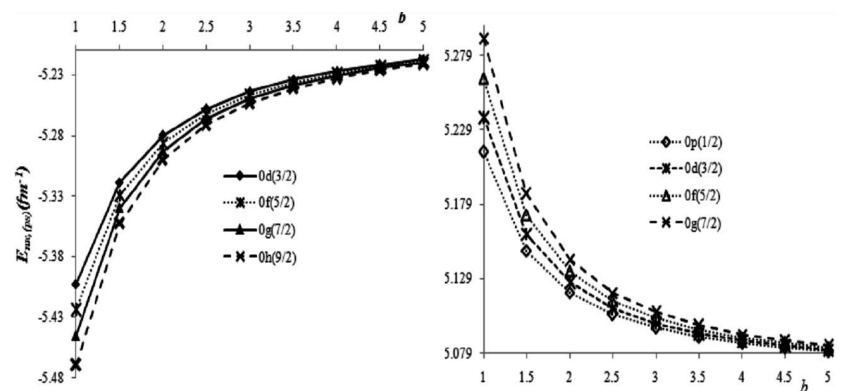

Fig. 5. Energy of the spin symmetry and pseudospin symmetry limits in the presence of Coulomb + Hulthen tensor interaction versus $b$.

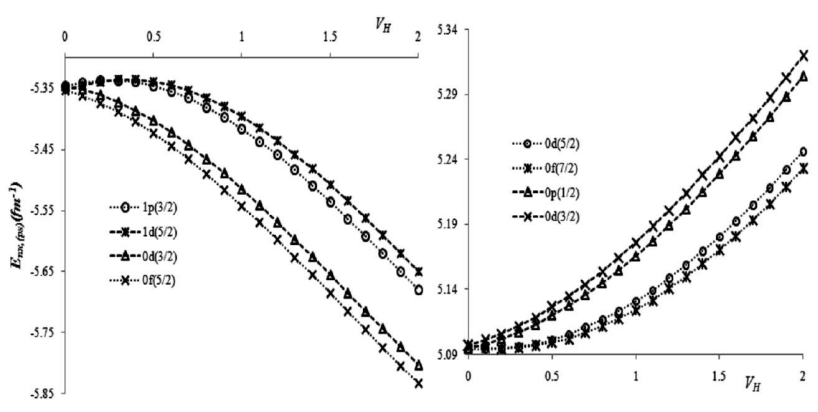

Fig. 6. Energy of the spin symmetry and pseudospin symmetry limits in the presence of Coulomb + Hulthen tensor interaction versus $V_{\mathrm{H}}$.

[4] H. Hassanabadi, B.H. Yazarloo, S. Zarrinkamar, H. Rahimov, Commun. Theor. Phys. 57, 339 (2012).

[5] S.H. Dong, Commun. Theor. Phys. 55, 969 (2011).

[6] H. Hassanabadi, B.H. Yazarloo, N. Salehi, J. Indian, Phys. 88, 405 (2014).

[7] E. Maghsoodi, H. Hassanabadi, S. Zarrinkamar, H. Rahimov, Phys. Scr. 85, 055007 (2012).

[8] H. Hassanabadi, B.H. Yazarloo, M. Mahmoudieh, S. Zarrinkamar, Eur. Phys. J. Plus 128, 111 (2013).

[9] N. Akpan, B.H. Ikot, S. Yazarloo, H. Zarrinkamar, Hassanabadi, Eur. Phys. J. Plus 129, 79 (2014).

[10] A.N. Ikot, Few-Body Syst. 53, 549 (2012).

[11] N. Akpan, H. Ikot, B.H. Hassanabadi, S. Yazarloo, Zarrinkamar, Int. J. Mod. Phys. E 22, 1350048 (2013).

[12] G.F. Wei, S.H. Dong, Europhys. Lett. 87, 40004 (2009).

[13] G.F. Wei, S.H. Dong, Phys. Lett. A 373, 49 (2008).

[14] G.F. Wei, S.H. Dong, Phys. Lett. A 373, 2428 (2009).

[15] G.F. Wei, S.H. Dong, Phys. Scr. 81, 035009 (2010).

[16] G.F. Wei, S.H. Dong, Phys. Lett. B 686, 288 (2010).

[17] G.F. Wei, S.H. Dong, Europhys. J. A 46, 207 (2010).

[18] G.F. Wei, S.H. Dong, Europhys. J. A 43, 185 (2010).

[19] J.N. Ginocchio, Phys. Rep. 414, 165 (2005).

[20] J.N. Ginocchio, Phys. Rev. C 69, 034318 (2004).

[21] J.N. Ginocchio, Phys. Rev. Lett. 78, 436 (1997).

[22] A. Bohr, I. Hamamoto, B.R. Mottelson, Phys. Scr. 26, 267 (1982) 
[23] D. Troltenier, C. Bahri, J.P. Draayer, Nucl. Phys. A 586, 53 (1995).

[24] P.R. Page, T. Goldman, J.N. Ginocchio, Phys. Rev. Lett. 66, 204 (2001).

[25] H. Hassanabadi, E. Maghsoodi, A.N. Ikot, S. Zarrinkamar, Appl. Math. Comp. $\mathbf{2 1 9}$ 9388 (2013).

[26] H. Hassanabadi, B.H. Yazarloo, L.L. Lu, Chin. Phys. Lett. 29, 020303 (2012).

[27] B.H. Yazarloo, H. Hassanabadi, S. Zarrinkamar, Eur. Phys. J. Plus 127, 51 (2012).

[28] A.F. Nikiforov, V.B. Uvarov, Special Functions of Mathematical Physics, Birkhauser, Basel 1988.

[29] M.G. Miranda, G. Huasun, S.H. Dong, Int. J. Mod. Phys. E 19, 123 (2010).

[30] M.C. Zhang, G.H. Sun, S.H. Dong, Phys. Lett. A 374, 704 (2010).
[31] C. Tezcan, R. Sever, Int. J. Theor. Phys. 48, 337 (2009).

[32] S. Zarrinkamar, S.F. Forouhandeh, B.H. Yazarloo, H. Hassanabadi, Eur. Phys. J. Plus 128, 109 (2013).

[33] H. Hassanabadi, S.F. Forouhandeh, H. Rahimov, S. Zarrinkamar, B.H. Yazarloo, Can. J. Phys. 90, 299 (2012)

[34] N. Ferkous, A. Bounames, Commun. Theor. Phys. 59, 679 (2013).

[35] W.C. Qiang, S.H. Dong, Phys. Lett. A 368, 13 (2007).

[36] S.H. Dong, W.C. Qiang, G.H. Sun, V.B. Bezerra, J. Phys. A 40, 10535 (2007). 